\title{
Role of Elastography in Assessment of Enlarged Lymph Nodes
}

\author{
Mohamed Zaki Ali Mourad, Nahla Mohamed Ali, Hala Mohammed Ahmed * \\ Department of Diagnostic Radiology, Faculty of Medicine - Sohag University
}

*Correspondence author: Hala Mohammed Ahmed

\begin{abstract}
Background: Enlarged lymph node is a marked dilemma in clinical practice. Common causes included; reactive hyperplasia, lymphoma, lymphadenitis, granuloma, metastasis, tuberculosis.

Objective: The aim of our study was to evaluate the diagnostic accuracy of shear wave elastography in differentiation between benign and malignant lymphadenopathy and to highlight the adjuvant rule of elastography as a complementary imaging technique to the conventional US.

Methodology: Our study included 25 patients who fulfilled the selection criteria and formed the study population. The present study was conducted in Radiology Department, Sohag University hospital from January 2017 till June 2017. The mean age was $46.4 \pm 17.1$ years ranged from 22 to 80 years. They were subjected to B-mode ultrasonography (US), Color Doppler sonography (CDS) and shear wave elastography.

Results: The optimal cut-off points were $19.44 \mathrm{Kpa}$ and $2.59 \mathrm{~m} / \mathrm{s}$ for average elasticity and mean shear wave velocity respectively. It shows $68.75 \%, 77.8 \%$ and $72 \%$ sensitivity, specificity, and accuracy respectively in the detection of malignant lymphadenopathy. The efficacy reached $92 \%, 100 \%$ and $93.8 \%$ for sensitivity, specificity, and accuracy respectively after exclusion of tuberculous and lymphoproliferative cases with significant improvement in efficacy of shear wave elastography.

Conclusion: CDS has more efficacy than the conventional US at detection of malignant lymphadenopathy and shear wave elastography has additive value for both.

Keywords: Ultrasound Elastography, Enlarged Lymph Nodes, US, CT.

Abbreviations: $\mathrm{ARFI}=$ acoustic radiation force impulse, $\mathrm{CDS}=$ Color Doppler sonography, $\mathrm{CT}=$ computed tomography, $\mathrm{EDV}=$ end diastolic velocity, $\mathrm{FNAB}=$ fine needle aspiration biopsy, $\mathrm{LNs}=$ lymph nodes, $\mathrm{MRI}=$ magnetic resonance imaging, $\mathrm{mSWV}=$ mean shear wave velocity, NHL= Non-Hodgkin lymphoma, $\mathrm{PI}=$ pulsitility index, $\mathrm{PS}=$ peak systolic velocity, $\mathrm{RI}=$ resistance index, $\mathrm{ROI}=$ region of interest, $\mathrm{S} / \mathrm{D}$ ratio $=$ systolic/diastolic ratio, $\mathrm{VTIQ}=$ virtual touch tissue imaging quantification, $\mathrm{UE}=$ ultrasound elastography.
\end{abstract}

\section{Introduction:}

Evaluation of lymphadenopathy has been made by various imaging modalities like; conventional ultrasonography (US), US-guided fineneedle aspiration cytology, computed tomography (CT) and magnetic resonance imaging (MRI) [1]. Ultrasound elastography (UE) is considered a new and non-invasive imaging technique used for the depiction of tissue hardness and elastic properties [2].

Shear wave elastography is a complementary imaging technique to the grey-scale US and could markedly reduce the unnecessary biopsies and is considered also a quantitative analytic method for elasticity using shear wave 
speed $(\mathrm{m} / \mathrm{s})$ or elastic modulus $(\mathrm{kPa})$ [3].

Acoustic radiation force impulse (ARFI) imaging, is a new elastography technique that reduces operator dependency and improves efficacy due to the addition of automated tissue compression. This technique uses focused high-intensity and rapid acoustic impulses from US transducer to form localized tissue displacements producing shear waves in the region of interest (ROI), which propagation that caused by the pushing pulse can be tracked providing a quantitative assessment of tissue stiffness. The more tissue stiffness, the more shear wave velocity it produces [1].

Virtual Touch tissue Imaging Quantification (VTIQ) is a new measurement technique which uses ARFI imaging technology to cause gentle tissue displacement for assessment of tissue stiffness in both qualitative and quantitative pattern. Multiple push pulses are used across the transducer face at multiple depths resulting in an elastogram of tissue stiffness which depends on the variation of shear wave velocity. Multiple ROIs can be placed on the elastogram to detect pulse sequences that can measure localized shear wave velocity from 0.5 to $10 \mathrm{~m} / \mathrm{s}$ in multiple locations [1].

The VTIQ image is a color-coded display of relative shear wave velocities within the user-defined ROI superimposed onto a conventional Bmode US image [4].

Many different types of organs can be evaluated by US elastography, including the breast، liver, prostate, thyroid glands, blood vessels, salivary glands, musculoskeletal structures, and lymph nodes. Its ability in the evaluation of lymphadenopathy, including a variety of benign and malignant pathologies has been discussed by several pilot studies.
However, these studies revealed marked selection bias since US elastography was applicated for pathologic verification or assessment of abnormal US findings, thereby resulting in samples included a high proportion of malignancies [5].

\section{Aim of the Work:}

The aim of our study is to evaluate the diagnostic accuracy of shear wave elastography in differentiation between benign and malignant causes of lymphadenopathy and to highlight the adjuvant rule of elastography as a complementary imaging technique to the conventional US.

\section{Patients and Methods:}

This study included 25 adult patients presented by enlarged lymph nodes in the period from January 2017 to June 2017. It was done according to the clinical research ethics of Sohag university hospital and the patients had given their written consent before entering the study.

Included patients were above 18 years presented with enlarged lymph nodes detected by US examination (cervical, axillary or inguinal) and are fit to undergo all protocol procedures, while the excluded patients were those who had the previous history of lymph node incisional biopsy or treated lymphadenopathy either medically or by radiotherapy over the region containing studied lymph nodes.

In addition to general contraindications to true cut needle biopsy such as ulcerated skin, suspected TB caseation, unstable heart diseases or sensitivity to local anesthesia.

All patients were subjected to Ultrasonographic examination including color-Doppler examination and shear wave elastography, a biopsy from the enlarged lymph nodes either True cut or excisional. If lymphadenopathy was highly 
suggested to be benign in nature according to clinical and US examination, follow up after 6 monthstherapeutic tests would be done.

Ultrasonography protocol:

All patients were examined using APLIO 500 TOSHIBA device at the Diagnostic radiology department of Sohag University Hospital.

1) B-mode: including number, size, shape, borders, long and short axis diameters, long to short axis ratio, echogenicity, presence of fatty hilum and other criteria as microcalcifications and cystic changes.

2) Color Doppler study: including estimation of peak systolic velocity (PSV), end-diastolic velocity (EDV) and resistance index (RI).

3) Power Doppler.

4) Elastography: After a dedicated sonographic examination.

Shear wave ultrasound elastography for the enlarged lymph nodes was performed with a linear-array transducer with a frequency range of $11-18 \mathrm{MHz}$ and the main elasticity values of the enlarged lymph nodes are obtained by KPa.

Our standard of reference was based on the histopathologic findings of a true cut needle or excisional biopsy from the enlarged lymph nodes or clinical and imaging follow up.

\section{Statistical analysis:}

Recorded data were analyzed using the statistical package for social sciences, version 20.0 (SPSS Inc., Chicago, Illinois, USA). Quantitative data were expressed as mean \pm standard deviation (SD). Qualitative data were expressed as frequency and percentage.

Sensitivity, specificity, positive predictive value, negative predictive value and accuracy of B-mode examination, colorDoppler study and ultrasound elastography were estimated for differentiation between benign and malignant enlarged lymph nodes.

\section{Results:}

This present study included 25 patients who fulfilled the selection criteria. Their ages ranged from 22 years to 80 years, divided like $12(48 \%)$ males and $13(52 \%)$ females.

Sixteen cases were finally diagnosed as malignant lymphadenopathy (ten cases were metastatic lymphadenopathy and 6 cases were lymphoproliferative disorders), while 9 cases were diagnosed as benign lymphadenopathy (Fig. 1-8).

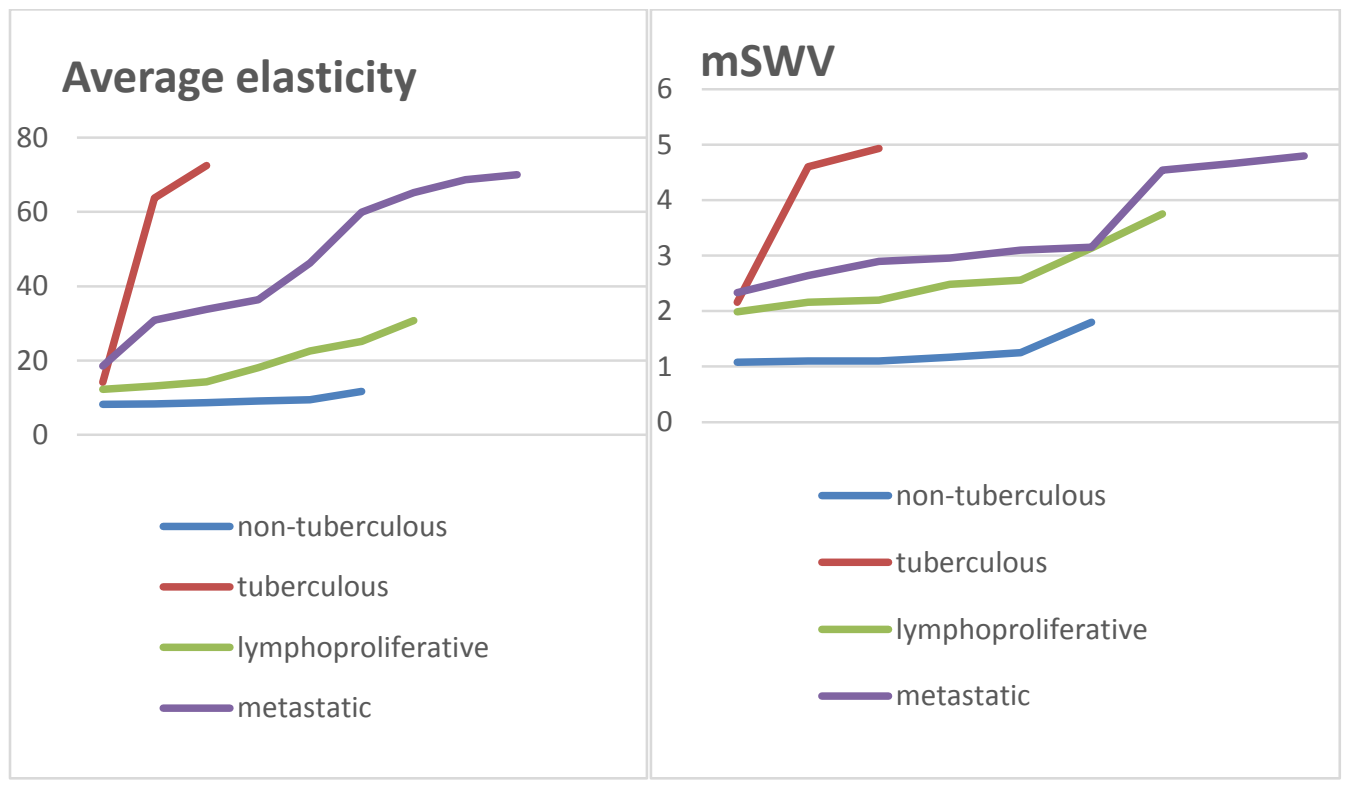


SOHAG MEDICAL JOURNAL Role of Elastography in Assessment of Enlarged Lymph Nodes Vol. 23 No. 3 July 2019 Hala Mohammed Ahmed

Fig. 1. Average elasticity and mSWV of examined L.N.s based on final diagnosis

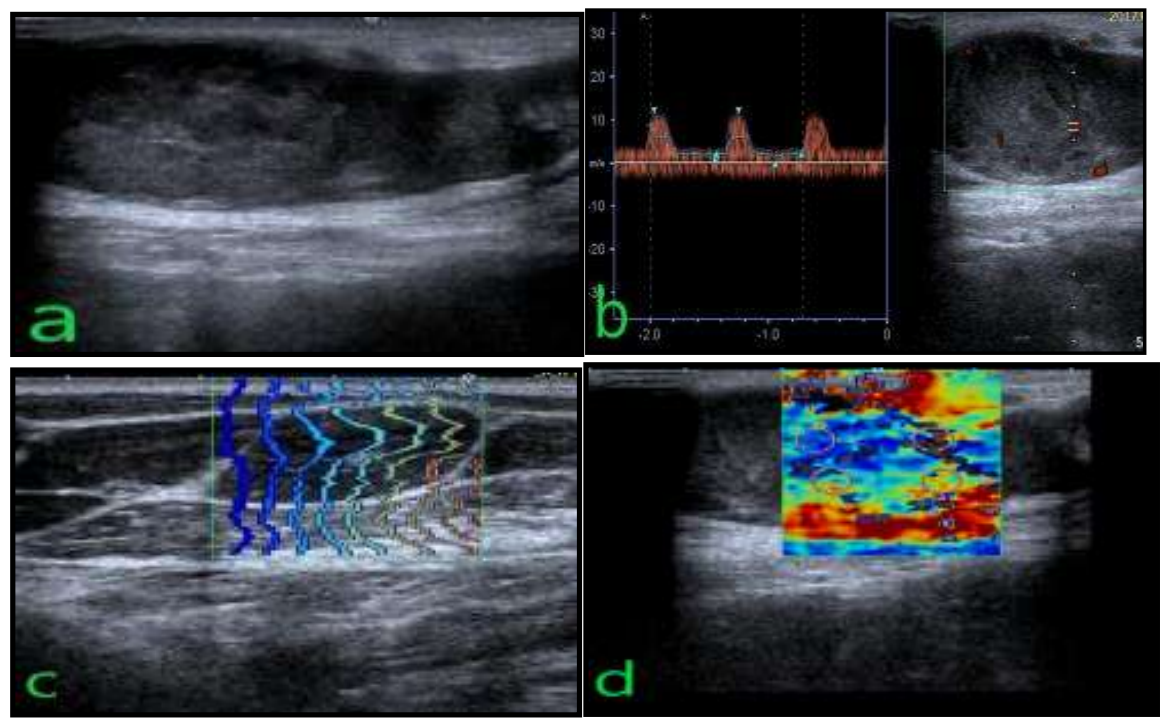

Fig. 2. 60 years old female patient presented by right inguinal metastatic lymphadenopathy of cancer urinary bladder treated by radical cystectomy

US examination of the predominant lymph node:

(a) B-mode; it measured $45 \times 22 \mathrm{~mm}$, oval-shaped, hypoechoic with lost hilum.

(b) CDS; resistance index (RI) was 0.77, pulsatility index (PI) was 1.88 and S/D ratio was 4.3.

(c) Color map.

(d) Shear wave elastography; its average elasticity was $16.7 \mathrm{KPa}$ and shear wave speed was 2.24 $\mathrm{m} / \mathrm{s}$.

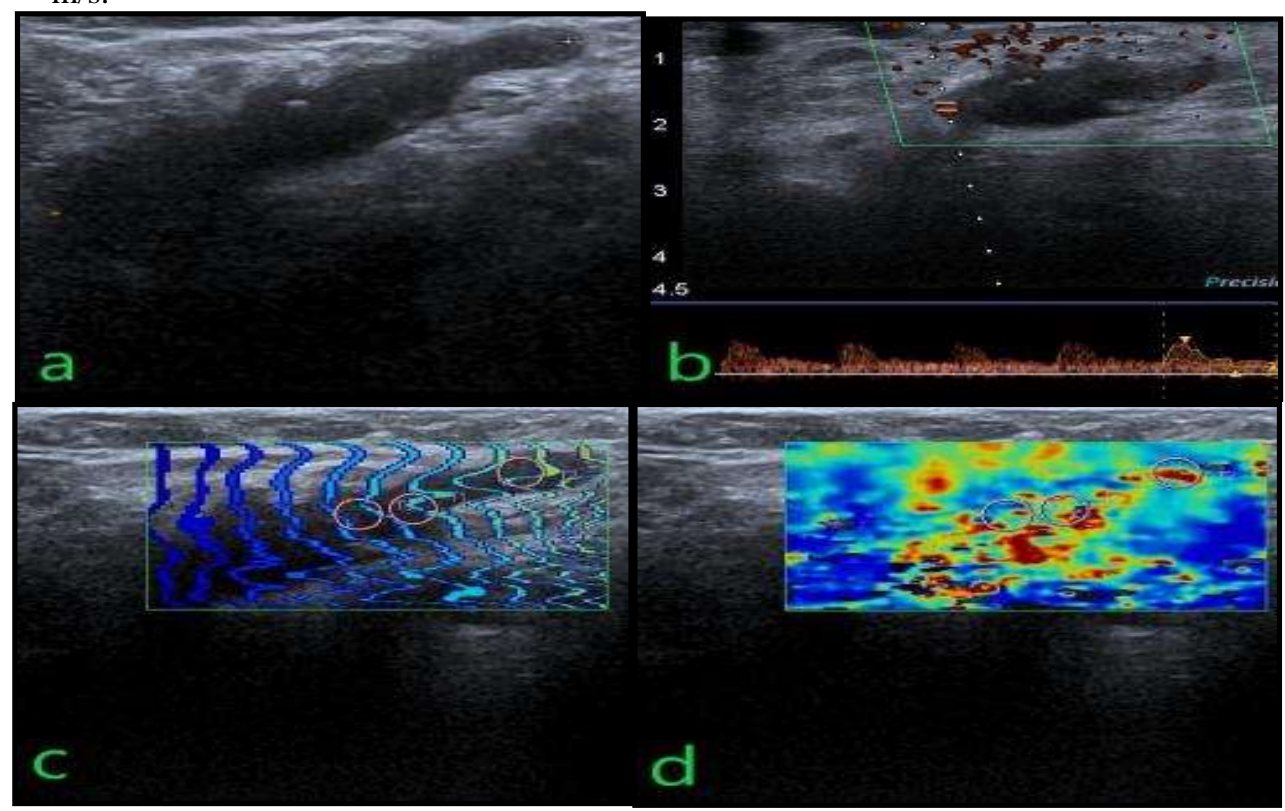

Fig. 3. 33 years old female patient presented by left axillary tuberculous lymphadenopathy past history of pulmonary tuberculosis followed by anti-TB treatment 5 years ago.

US examination of the predominant lymph node:

(a) B-mode; it measured $5 \times 1.2 \mathrm{~cm}$, oval in shape, hypoechoic with irregular outline and lost hilum.

(b) CDS; resistance index (RI) was 3.73, pulsatility index (PI) was 1.41 and S/D ratio was 1.56 .

(c) Color map.

(d) Shear wave elastography; average elasticity was $63.77 \mathrm{KPa}$ and shear wave speed was 4.6 $\mathrm{m} / \mathrm{s}$. 
SOHAG MEDICAL JOURNAL Role of Elastography in Assessment of Enlarged Lymph Nodes
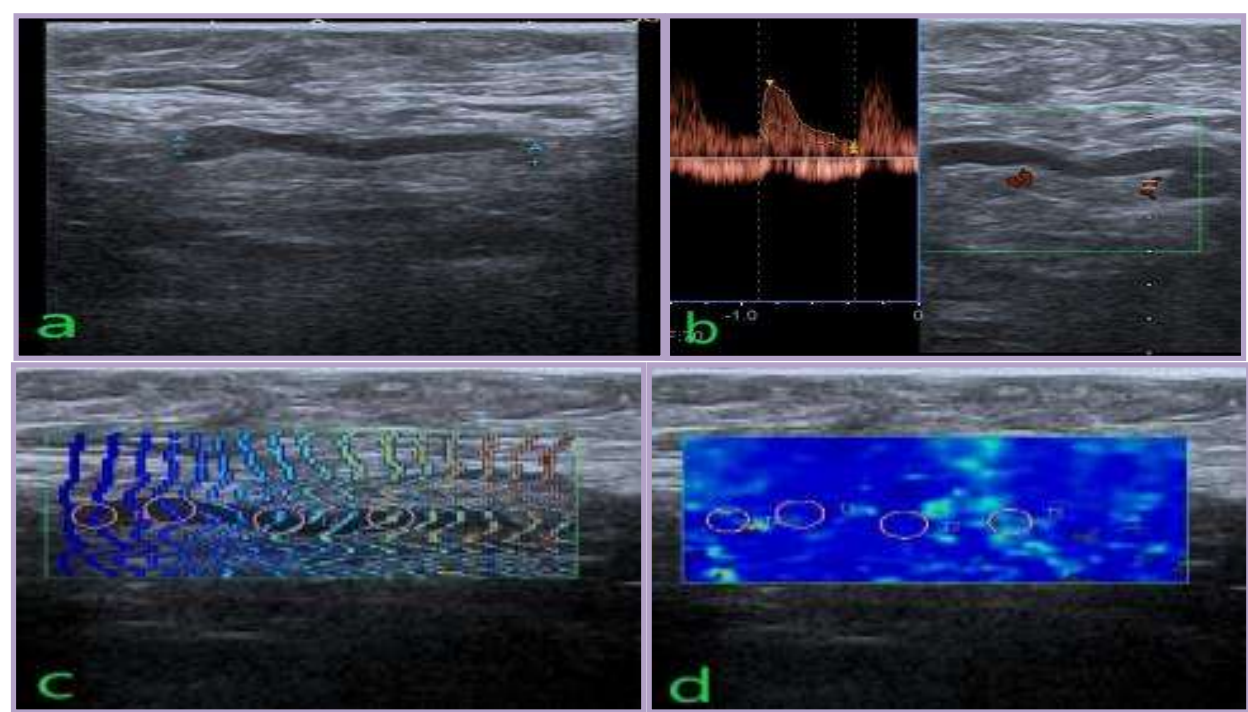

Fig. 4. $\quad 60$ years old male patient presented by cervical, axillary and inguinal chronic leukemic lymphadenopathy.

US examination of right axillary enlarged L.N.:

(a) B-mode; it measured $3.3 \times 1.2 \mathrm{~cm}$, oval in shape, isoechoic, well-circumscribed with preserved hilum.

(b) CDS; resistance index (RI) was 0.78, pulsatility index (PI) was 1.69 and S/D ratio was 4.47.

(c) Color map.

(d) Shear wave elastography; average elasticity was $18.1 \mathrm{KPa}$ and shear wave speed was $2.56 \mathrm{~m} / \mathrm{s}$.
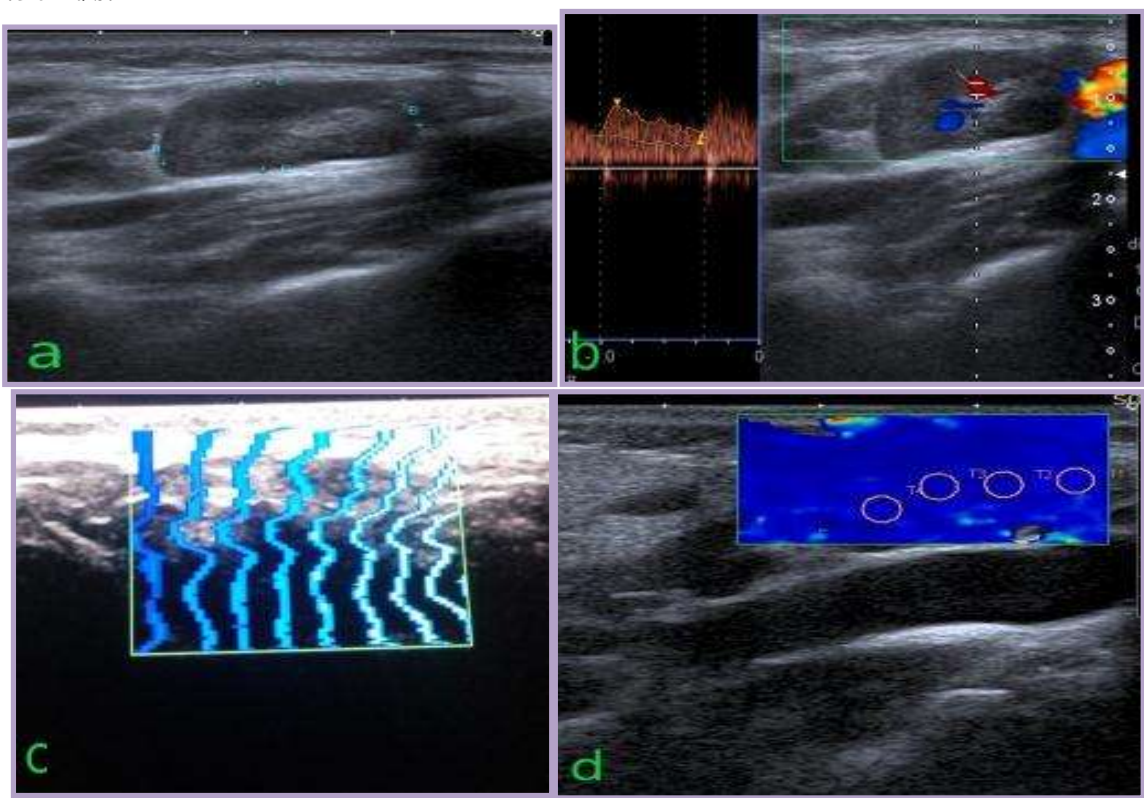

Fig. 5. 30 years old female patient had Ludwig's angina and presented by bilateral submandibular reactive lymphadenopathy.

US examination of a predominant lymph node:

(a) B-mode; it measured $20 \times 9 \mathrm{~mm}$, isoechoic pattern, oval-shaped, well-circumscribed with a regular outline and preserved hilum.

(b) CDS; resistance index (RI) was 0.25 , pulsatility index (PI) was 0.68 and S/D ratio was 1.34 .

(c) Color map.

(d) Shear wave elastography; average elasticity was $9.37 \mathrm{KPa}$ and shear wave speed was $1.1 \mathrm{~m} / \mathrm{s}$. 
SOHAG MEDICAL JOURNAL Role of Elastography in Assessment of Enlarged Lymph Nodes
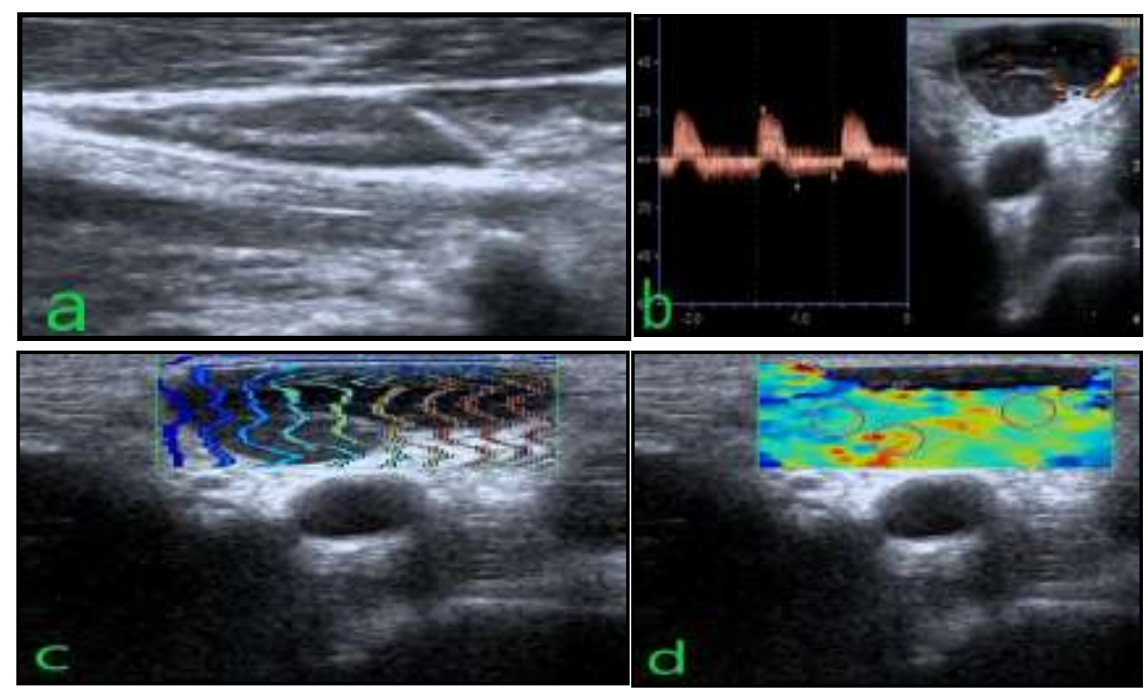

Fig. 6. 47 years old male patient had NHL presented by cervical, axillary and inguinal lymphadenopathy.

US examination showed:

(a) B-mode; Multiple enlarged bilateral deep cervical, axillary and inguinal lymph nodes ranging from $10 \times 7 \mathrm{~mm}$ up to $22 \times 9 \mathrm{~mm}$ with more or less oval in shape, isoechoic pattern and preserved hila.

(b) CDS; resistance index (RI) was 0.36, pulsatility index (PI) was 0.84 and S/D ratio was 1.56 .

(c) Color map.

(d) Shear wave elastography; average elasticity was $14.4 \mathrm{KPa}$ and shear wave speed was 2.16 $\mathrm{m} / \mathrm{s}$.
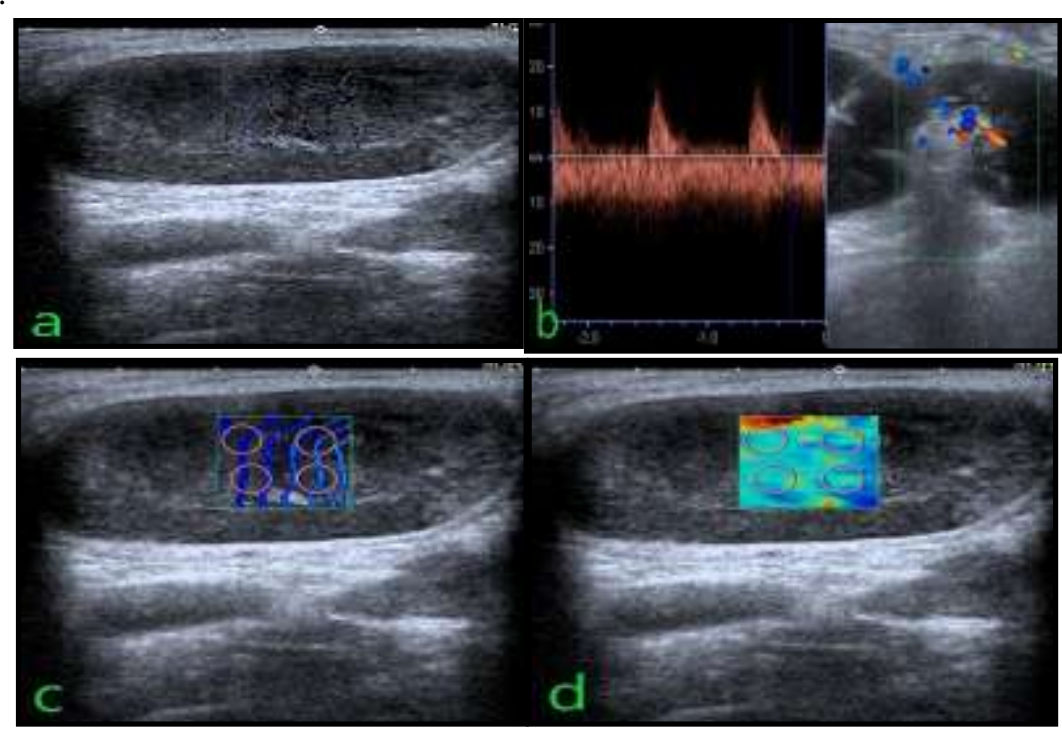

Fig. 7. 65 years old female patient known as cancer mandible and presented by metastatic cervical lymphadenopathy.

US examination of the largest lymph node seen submental:

(a) B-mode; it measured $5.3 \times 3.2 \mathrm{~cm}$ with oval shape, isoechoic pattern and lost hilum.

(b) CDS showed resistance index (RI) was 0.75, pulsatility index (PI) was 1.52 and the S/D ratio was 4.1 .

(c) Color map. 
(d) Shear wave elastography; its average elasticity was $30.82 \mathrm{KPa}$ and shear wave speed was $3.1 \mathrm{~m} / \mathrm{s}$.
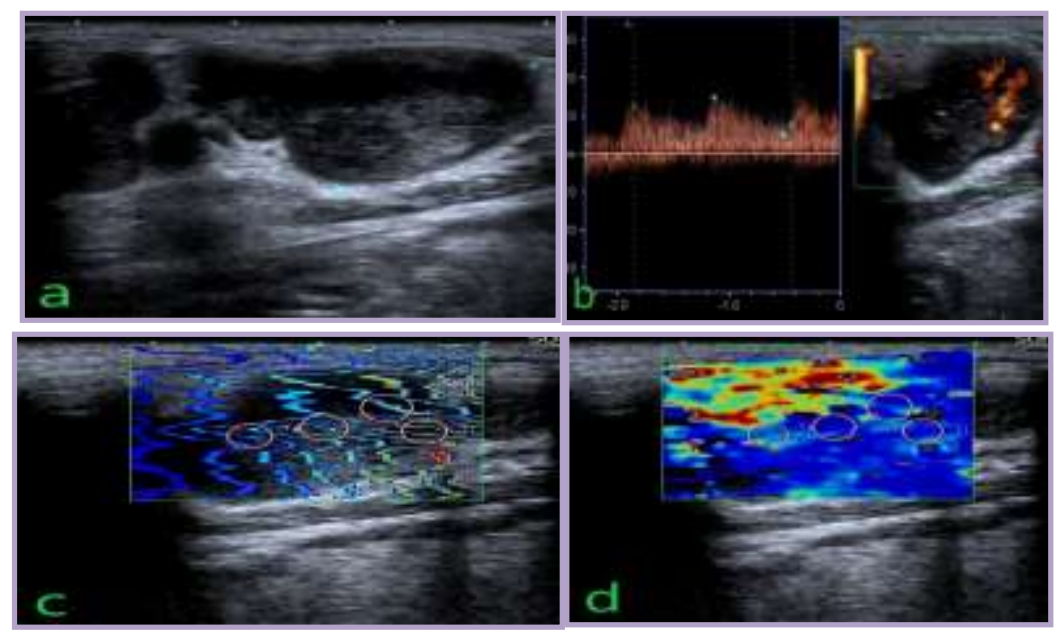

Fig. 8. 22 years old female patient was diagnosed as chronic parotitis and presented by right infra parotid reactive lymphadenopathy.

US examination of the predominant lymph node:

(a) B-mode, it measured $22 \times 15 \mathrm{~mm}$ with a hypoechoic pattern, oval shape, and preserved hilum.

(b) CDS; resistance index (RI) was 0.4 , pulsatility index (PI) was 0.72 and $S / D$ ratio was 3.87 .

(c) Color map.

(d) Shear wave elastography; average elasticity was $23.73 \mathrm{KPa}$ and shear wave speed was $2.64 \mathrm{~m} / \mathrm{s}$.

\section{Ultrasonographic findings:}

The mean size of the examined lymph nodes was 33.16

$\pm 12.06 \mathrm{~mm}^{3}$ ranged from $20 \mathrm{~mm}^{3}$ to 60 $\mathrm{mm}^{3}$.

Regarding B-mode ultrasonographic findings, the hypoechoic pattern, L/S ratio less than 2 and the lost fatty hilum were more significantly detected in malignant rather than benign lymphadenopathy with accuracy in diagnosis of $64 \%, 68 \%$ and $72 \%$, sensitivity of $88.9 \%, 68.8 \%$ and $62.5 \%$ and specificity of $50 \%, 66.7 \%$ and $88.9 \%$ respectively (Table 1).

\begin{tabular}{|c|c|c|c|c|c|}
\hline B-mode criteria & $\begin{array}{c}\text { Sensitivit } \\
\mathrm{y}(\%)\end{array}$ & $\begin{array}{c}\text { Specificit } \\
\mathrm{y}(\%)\end{array}$ & $\begin{array}{c}\text { Positive } \\
\text { predictive value } \\
(\%)\end{array}$ & $\begin{array}{c}\text { Negative } \\
\text { predictive } \\
\text { value }(\%)\end{array}$ & $\begin{array}{c}\text { Accuracy } \\
(\%)\end{array}$ \\
\hline Echopattern & 88.9 & 50 & 50 & 88.9 & 64 \\
\hline $\begin{array}{c}\text { Long axis /short axis } \\
\text { ratio (L/S ratio) }\end{array}$ & 68.8 & 66.7 & 78.7 & 54.5 & 68 \\
\hline Fatty hilum & 62.5 & 88.9 & 90.9 & 57.1 & 72 \\
\hline Overall efficacy & 73.4 & 68.53 & 73.2 & 66.83 & 68 \\
\hline
\end{tabular}

Table 1: Efficacy of B- mode US examination for the diagnosis of malignant lymphadenopathy

Considering the color-Doppler examination, the $S / D$ ratio was the most accurate parameter in the diagnosis of malignant lymphadenopathy (88\%) followed by the resistance index $(80 \%)$ and lastly the pulsatility index (72\%) 
with an overall accuracy of color-

(Table 2).

Doppler examination reaching $80 \%$

\begin{tabular}{|c|c|c|c|c|c|}
\hline $\begin{array}{c}\text { B-mode } \\
\text { criteria }\end{array}$ & $\begin{array}{c}\text { Sensitivity } \\
(\%)\end{array}$ & $\begin{array}{l}\text { Specificity } \\
(\%)\end{array}$ & $\begin{array}{c}\text { Positive } \\
\text { predictive } \\
\text { value }\end{array}$ & $\begin{array}{c}\text { Negative } \\
\text { predictive } \\
\text { value }\end{array}$ & $\begin{array}{c}\text { Accuracy } \\
(\%)\end{array}$ \\
\hline RI & 100 & 64.28 & 68.75 & 100 & 80 \\
\hline PI & 84.62 & 58.33 & 68.75 & 77.78 & 72 \\
\hline S/D ratio & 88.24 & 58.33 & 93.75 & 77.78 & 88 \\
\hline $\begin{array}{c}\text { Overall } \\
\text { efficacy }\end{array}$ & 90.95 & 60.31 & 77.08 & 85.19 & 80 \\
\hline
\end{tabular}

Table 2: Efficacy of CDUS examination for the diagnosis of malignant lymphadenopathy

In this study, the efficacy of shear wave elastography in differentiation between benign and malignant lymph nodes were based on average elasticity and SWV at different cut-off points and revealed that the results based on average elasticity showed higher sensitivity, PPV and NPV at cut-off point of $19.44 \mathrm{Kpa}$ than $30.2 \mathrm{Kpa}$, while the results based on SWV showed higher efficacy at cut-off point of 2.59 $\mathrm{m} / \mathrm{s}$ than $1.9 \mathrm{~m} / \mathrm{s}$ and $2.93 \mathrm{~m} / \mathrm{s}$.no change in specificity at different cut-off points either as regard $\mathrm{mSWV}$ or average elasticity of examined L.N.s (Table 3).

\begin{tabular}{|c|c|c|c|c|c|c|}
\hline $\begin{array}{c}\text { Shear wave elastography } \\
\text { parameters }\end{array}$ & $\begin{array}{l}\text { Cut-off } \\
\text { points }\end{array}$ & $\begin{array}{c}\text { Sensitivity } \\
(\%)\end{array}$ & $\begin{array}{l}\text { Specificity } \\
(\%)\end{array}$ & $\begin{array}{c}\text { Positive } \\
\text { predictive } \\
\text { value }\end{array}$ & $\begin{array}{c}\text { Negative } \\
\text { predictive } \\
\text { value }\end{array}$ & $\begin{array}{c}\text { Accuracy } \\
(\%)\end{array}$ \\
\hline \multirow[t]{2}{*}{ Average elasticity (Kpa) } & 19.44 & 68.75 & 77.78 & 84.62 & 58.33 & 72 \\
\hline & 30.2 & 56.25 & 77.78 & 56.25 & 50 & 64 \\
\hline \multirow{3}{*}{ SWV (m/s) } & 1.9 & 56.25 & 77.78 & 56.25 & 50 & 64 \\
\hline & 2.59 & 68.75 & 77.78 & 84.62 & 58.33 & 72 \\
\hline & 2.93 & 56.25 & 77.78 & 56.25 & 50 & 64 \\
\hline
\end{tabular}

Table 3: Efficacy of shear wave elastography at different cut-off points

On the basis of the final diagnosis, shear wave elastography results including average elasticity by Kpa and $\mathrm{SWV}$ by $\mathrm{m} / \mathrm{s}$ revealed that:

a. The tuberculous group showed SD of $\mathrm{mSWV}$ and average elasticity equals 1.51 and 31.48 respectively indicating high variation in results.

b. The results of the tuberculous group approach that of the metastatic group

as regards both mean elasticity and mSWV. c. Easier differentiation among different groups was established at

cut-off points of $19.44 \mathrm{Kpa}$ and $2.59 \mathrm{~m} / \mathrm{s}$ regarding average elasticity and $\mathrm{mSWV}$ respectively which showed higher sensitivity than other cut-off points.

Lymphoproliferative lymphadenopathy couldn't be distinguished from benign non-tuberculous lymphadenopathy due to the preservation of nodal architecture and the absence of extensive destruction seen at metastatic lymphadenopathy. Also, difficult differentiation between 
tuberculous and metastatic lymphadenopathy as nodal hardness increases in both. So, reassessment of the examined cases was done after exclusion of tuberculous and lymphoproliferative cases based on the most sensitive cut-off points $(19.44 \mathrm{Kpa}$ and $2.59 \mathrm{~m} / \mathrm{s}$ ) resulting in significant increase in efficiency of shear wave elastography in detecting metastatic lymphadenopathy $(92 \%, 100 \%$ and
93.75\% as regard sensitivity, specificity and accuracy respectively).

Lastly, comparison between the diagnostic performance of B-mode US, CDS and shear wave elastography after exclusion of tuberculous and lymphoproliferative cases revealed gradual increase at the parameters consequently except for the specificity which shows better value as regard Bmode US over CDS (68.53\% over $60.31 \%$ ) with the predominance for elastography (100\%) (Table4).

\begin{tabular}{|c|c|c|c|c|c|}
\hline Item & $\begin{array}{c}\text { Sensitivity } \\
(\boldsymbol{\%})\end{array}$ & Specificity (\%) & PPV (\%) & NPV (\%) & Accuracy (\%) \\
\hline B-mode US & 73.4 & 68.53 & 73.2 & 66.83 & 68 \\
\hline CDS & 90.95 & 60.31 & 77.08 & 85.19 & 80 \\
\hline Shear wave elastography & 92 & 100 & 100 & 85.71 & 93.75 \\
\hline
\end{tabular}

Table 4: Comparison between the diagnostic performance of B-mode sonography, colorDoppler study and shear wave elastography of scanned LNs after exclusion of tuberculous and lymphoproliferative cases

\section{Discussion:}

Our study was conducted upon 25 cases of superficial lymphadenopathy proven by preliminary US exami-nation. The mean of the age of patients was $46.4 \pm$ 17.1 years, ranged from 22 to 80 years.

By using the B-mode ultrasound examination and as regards the echopattern of examined lymph nodes; 8 cases of the 16 proven malignant cases (50\%) showed hypoechoic echopattern. Only 3 of the other 8 cases that had isoechoic pattern were metastatic (two cases had papillary thyroid carcinoma and one case had cancer breast). The other 5 were of lymphoproliferative diseases.

This agrees with Özel et al. [6] who reported that metastatic nodes tend to be hypoechoic compared with adjacent muscles. However, metastatic nodes from the papillary carcinoma of the thyroid gland are usually hyperechoic. This hyperechogenicity of lymph nodes is believed to result from the intranodal deposition of thyroglobulin, which originates from the thyroid primary tumor.

While eight cases of the 9 proven benign cases $(88.9 \%)$ had isoechoic echopattern and the last one that had hypoechoic echopattern was previously treated recurrent TB lymphadenitis which might have a role in the resultant echopattern of examined lymph nodes.

As regards the long/short diameter ratio (L/S ratio); 11 malignant cases $(68.8 \%)$ showed an L/S ratio of less than 2 , and 4 of the other 5 proven malignant cases that had an L/S ratio more than 2 were lymphoproliferative diseases. The other one was a case of cancer mandible. so, after exclusion of cases with lymphoproliferative diseases, 8 cases of the 9 proven metastatic lymph nodes $(88.9 \%)$ had $\mathrm{L} / \mathrm{S}$ ratio less than 2 . While six cases of the proven benign cases had an L/S ratio equals or more than 2 . The other 3 
were two tuberculous and one reactive for Ludwig's angina.

As regards the presence of fatty hilum; 10 cases $(62.5 \%)$ showed lost hila. 4 of the other 6 cases that had preserved hila were lymphoproliferative diseases and the other two were thyroid adenocarcinoma and breast cancer. so, after the exclusion of cases with lymphoproliferative diseases, 7 cases of the 9 proven metastatic lymph nodes $(77.8 \%)$ had lost hila.

Eight cases of proven benign cases had preserved hila. The last one was tuberculous.

Our final results of B-mode efficacy in differentiation between benign and malignant lymphadenopathy revealed $68.8 \%$ sensitivity, $66.7 \%$ specificity and $68 \%$ accuracy for the criterion of $\mathrm{L} / \mathrm{S}$ ratio at a cut off value of 2 and $62.5 \%$ sensitivity, $88.9 \%$ specificity, and $72 \%$ accuracy as regard the presence of fatty hilum.

Our results support the findings of Özel et al. [6]. That study declared sensitivities of $65-68.3 \%$ and specificities of $77 \%-80.6 \%$ for $\mathrm{L} / \mathrm{S}$ ratio and lost fatty hilum respectively.

Our results relatively agree with a study by Jukuri et al. [7] which included 72 patients ranged in age between 18-75 years. That study published the individual criteria for malignancy on ultrasound and color Doppler (didn't include the criterion of nodal echopattern) compared with the histopathology and reported that 20 cases had L/S ratio less than 2 of the 22 proven malignant cases $(90.9 \%)$ and 19 cases had lost hila of the 22 proven malignant cases $(86.3 \%)$. It didn't include cases with lymphoproliferative diseases

By using color Doppler examination; 11 cases of the 16 proven malignant cases $(68.8 \%)$ showed resistance index more than or equals 0.8 and 11 cases $(68.8 \%)$ showed pulsatility index equals or more than 1.4 while 15 cases
(93.75\%) showed S/D ratio more than 2 . overall cases that matched the final diagnosis were 20, 18 and 22 cases as regards the previous parameters with percentage of $80 \%, 72 \%$ and $88 \%$ of the examined group respectively.

All proven benign cases had a resistance index of less than 0.8 . While two cases had escaped the results for benign lymphadenopathy considering pulsatility index and S/D ratio; both of them were tuberculous.

Similarly to our study, a conducted study from India was performed on 80 lymph nodes from 72 patients of which 8 were known cases of head and neck carcinomas who were evaluated with ultrasound and color Doppler Jukuri et al. [7] and cancer detection rate was of $86 \%$ and $82 \%$ using the resistance index (RI) and pulsatility index (PI) at cut-off points of 0.8 and 1.4 consequently. It has to be considered, however, that the parameter of the S/D ratio wasn't used in evaluation of those cases as a part of color Doppler examination.

According to Ahuja et al. [8], the reason for the contrasting results of the values of RI and PI is the differences in methodology and vessel sampling used for evaluating the RI and PI, such as which node should be evaluated and how the RI and PI are calculated.

We reported $90.95 \%, 60.31 \%$ and $80 \%$ sensitivity, specificity, and accuracy for $\mathrm{CD}$ examination in detecting malignant lymph nodes.

Ying et al. [9] reported that Doppler ultrasonography evaluation of nodal vascular pattern has high sensitivity (83-89\%) and specificity (87-100\%) for differentiating metastatic and reactive nodes. While in distinguishing lymphomatous and reactive nodes, the sensitivity and specificity are $67 \%$ and $100 \%$, respectively.

By using shear wave elastography and as regard average elasticity of examined tissues; 9 and 11 cases were detected as malignant at 30.2 and 19.44 Kpa cut-off 
points representing $56.3 \%$ and $68.8 \%$ of malignant cases respectively. A cut-off point of $30.2 \mathrm{Kpa}, 5$ of the other 6 cases were lymphoproliferative diseases and the last case was breast cancer but at a cut-off point of $19.44 \mathrm{Kpa}, 4$ of the other 5 cases were lymphoproliferative diseases and the last case was the same case of breast cancer.

As regards the benign cases; two cases with tuberculous lymphadenopathy had escaped benign results and had average elasticity of examined lymph nodes higher than $30.2 \mathrm{Kpa}$ and $\mathrm{mSWV}$ higher than $2.93 \mathrm{~m} / \mathrm{s}$.

It means that L.N.s affected by lymphoproliferative diseases tends to approach that of benign ones. That agrees with Tang et al. [10] which reported that lymphomatous nodes tend to be softer than metastatic nodes and have a similar elastographic score to benign nodes. Therefore, lymphomatous and benign nodes could not be distinguished according to the elastographic score alone.

Also, unclear differentiation between tuberculous and metastatic lymphadenopathy is thought to refer to the destructive pattern of TB and the formation of excessive fibrosis leading to increased nodal stiffness.

After the exclusion of lymphoproliferative and tuberculous cases, we reached $88.8 \%$ cancer detection rate of metastatic L.N.s and $92 \%, 100 \%$ and $93.75 \%$ for sensitivity, specificity, and accuracy respectively at $19.44 \mathrm{Kpa}$ and $2.59 \mathrm{~m} / \mathrm{s}$. This agrees with Choi et al. [11] that reported $91 \%$ cancer detection rate of metastatic cervical lymphadenopathy at $19.44 \mathrm{Kpa}$ cut-off point as $\mathrm{mSWV}$. While, Bhatia et al. [12] reported $41 \%$ cancer detection rate as regard cervical lymphadenopathy at $30.2 \mathrm{Kpa}$ cut-off point as $\mathrm{mSWV}$ and this supports our results when $19.44 \mathrm{Kpa}$ is more sensitive than $30.2 \mathrm{Kpa}$ as cut-off point for average elasticity in cancer detection and disagree with Ying et al. [9] who reported that optimal cut-off of tissue stiffness was $30.2 \mathrm{kPa}$ with sensitivity, specificity, and accuracy of $41.9 \%$, $100 \%$, and $61.8 \%$, respectively.

There are relatively few clinical studies that have compared acoustic radiation force impulse imaging and supersonic shear imaging [5, 12, 13]. Bhatia et al. [12] have reported that the median elastic modulus of malignant lymph nodes is higher than that of benign lymph nodes. However, discrimination was low because the optimal cut-off value of $30.2 \mathrm{kPa}$ demonstrated sensitivity, specificity, and accuracy values of $41.9 \%, 100 \%$, and $61.8 \%$, respectively. Another study Choi et al. [5] has reported that the maximum elastic modulus can be used to differentiate malignant lymph nodes and that a cut-off value of $19.4 \mathrm{kPa}$ resulted in accuracy, sensitivity, and specificity values of $94 \%, 91 \%$, and $97 \%$, respectively.

As regards the mean shear wave velocity at examined lymph nodes (mSWV); 11 cases were detected as malignant at $2.59 \mathrm{~m} / \mathrm{s}$ cut-off point $(68.8 \%)$ and 9 cases were detected as malignant at both 1.9 and $2.93 \mathrm{~m} / \mathrm{s}$ cutoff points representing $56.3 \%$ of malignant cases.

Fujiwara et al. [14] reported that 19 malignant cervical lymph nodes could be detected from the total of 20 malignant lymph nodes $(95 \%)$ at 19 patients ranged from 23 up to 85 years old using $1.9 \mathrm{~m} / \mathrm{s}$ as cut-off value mean shear wave velocity (mSWV). The final diagnosis was based on excisional biopsy after neck dissection. However, Meng et al. [13] estimated the cut-off level of virtual touch tissue quantification value at $2.59 \mathrm{~m} / \mathrm{s}$ and could confirm malignancy at 78 cervical lymph nodes from 94 as a total number of malignant cervical lymph nodes proved histological by means of surgery, biopsy and FNA cytology. 
Choi et al. [5] had a recent systematic review and meta-analysis that was published and detailed the currently available techniques for assessing the enlarged lymph nodes and described the main published results, reproducibility and limitations of the US elastography in clinical practice. Till its date, many studies have reported various sensitivity and specificity values for the efficacy of shear wave elastography with or without one or more additional imaging techniques for differentiation between benign and malignant lymph nodes. More than 10 pilot studies have been published on the use of US elastography to evaluate the cervical lymph nodes.

\section{Conclusion:}

- CDS has more efficacy than the conventional US at detection of malignant lymph nodes and shear wave elastography has additive value for both.

- Shear wave elastography shows high specificity and low sensitivity, thus it's not suitable as a screening tool for nodal malignancy.

- The advantages of shear wave elastography include the absence of ionizing radiation, easy performance, not time-consuming, non-invasive, low cost, bedside availability, high specificity in nodal malignancy.

- Similar elastography findings of lymphoproliferative L.N.s with benign L.N.s and of tuberculous L.N.s with metastatic L.N.s should be considered to reach the optimal results.

\section{Recommendations:}

We recommend a large sample size with different pathologies as much as possible. This would have many accurate results. An examination can include all enlarged lymph nodes with their final diagnosis depending on FNAB which is less affected by nodal size. It is also more simple and easier to be performed. This would add more increase in sample size.

\section{References:}

1. Cheng KL, Choi YJ, Shim WH, Lee JH, and Baek JH. Virtual Touch Tissue Imaging Quantification Shear Wave Elastography: Prospective Assessment of Cervical Lymph Nodes. Ultrasound in medicine \& biology. 2016; 42:378-86.

2. Altahhan KN, Wang $Y$, Sobh $\mathbf{N}$, and Insana MF. Indentation Measurements to Validate Dynamic Elasticity Imaging Methods. Ultrasonic imaging 2016; 38(5): 332-45.

3. Suh CH, Choi YJ, Baek JH, Lee JH. The diagnostic performance of shear wave elastography for malignant cervical lymph nodes: A systematic review and meta-analysis. Eur Radiol. 2017; 27(1):222-230.

4. Azizi G, Keller JM, Mayo ML, Piper K, Puett D, Earp KM, and Malchoff CD. Shear Wave Elastography and Cervical Lymph Nodes: Predicting Malignancy. Ultrasound in Medicine \& Biology, 2016; 42:6:1273-81.

5. Choi YJ, Lee JH, and Baek JH. Ultrasound elastography for evaluation of cervical lymph nodes. Ultrasonography. 2015;34(3):157-64.

6. Özel D and Özel BD. Evaluation of the diagnostic value of conventional and color Doppler ultrasound with elastography strain ratios in differentiation between benign and malignant lymph nodes. Polish Journal of Radiology. 2018; 83(e):32-36.

7. Jukuri N, Narra R, Kuppili MM, Nalubolu S and Pasupuleti B. Role of ultrasound and color Doppler in the evaluation of cervical lymphadenopathy. 2015; 4:4:520-6.

8. Ahuja A, Ying $M$, Yuen $Y H$, and Metreweli C. Power Doppler sonography to differentiate tuberculous cervical lymphadenopathy from nasopharyngeal carcinoma. American Journal of Neuroradiology 2001; 22(4):735-40.

9. Ying M, Bhatia KS, Lee YP, Yuen HY, and Ahuja AT. Review of ultrasonography of malignant neck nodes: greyscale, Doppler, contrast 
enhancement and elastography. International Cancer Imaging Society. 2013; 13(4):658-99.

10.Tang A, Cloutier G, Szeverenyi NM, and Sirlin CB. Ultrasound Elastography and MR Elastography for Assessing Liver Fibrosis: Part 2, Diagnostic Performance, Confounders, and Future Directions. American journal of roentgenology. 2015; 205:33-40.

11.Choi YJ, Lee JH, Lim HK, Kim SY, Han MY, and Cho KJ. Quantitative Shear Wave Elastography In the Evaluation of Metastatic Cervical Lymph Nodes. Ultrasound in Medicine \& Biology. 2013; 39:6: 935-40.
12.Bhatia KS, Cho CC, Tong CS, Yuen EH, and Ahuja AT. Shear wave elasticity imaging of cervical lymph nodes. Ultrasound in medicine \& biology 2012; 38:195-201.

13.Meng $W$, Xing $P$, Chen $Q$ and $W u ~ C$. Initial experience of acoustic radiation force impulse ultrasound imaging of cervical lymph nodes. European Journal of Radiology. 2013; 82:1788-92.

14.Fujiwara T, Tomokuni J, Iwanaga $K$, Ooba S and Huji T. Acoustic Radiation Force Impulse Imaging for Reactive and Malignant/Metastatic Cervical Lymph Nodes. Ultrasound in Medicine \& Biology. 2013; 39(7): 1178-83. 
SOHAG MEDICAL JOURNAL Role of Elastography in Assessment of Enlarged Lymph Nodes 